\title{
New Measuring Method for Colorless Liquid Level Based on CLC100 Level Sensor and Powercast Wireless Sensor Kit
}

\author{
Guanlin Yan 1,2, Gerhard Lindner², Yuming Shen' \\ ${ }^{1}$ Department of Control Science and Engineering, University of Shanghai for Science and Technology, Shanghai, \\ China \\ ${ }^{2}$ Institute of Sensor and Actuator Technology, Coburg, Germany \\ Email: ygl effort@163.com, gerhard.lindner@hs-coburg.de, ym-shem@163.com
}

Received 9 February 2015; accepted 1 April 2015; published 3 April 2015

Copyright (C) 2015 by authors and Scientific Research Publishing Inc.

This work is licensed under the Creative Commons Attribution International License (CC BY). http://creativecommons.org/licenses/by/4.0/

(c) $\underset{\mathrm{BY}}{\mathrm{B}}$ Open Access

\section{Abstract}

In this paper, a new wireless measuring method for colorless liquid level measurement was presented, based on latest CLC100 liquid level sensor and Powercast wireless sensor development kit. The wireless system is divided into two parts, level measurement and data transmission part as well as data receiving and display part. First part included the capacitive liquid level sensor CLC100 and the wireless senor board. CLC100 sensor was used for liquid level measurement. Wireless sensor kit from Powercast Corporation included one wireless sensor board, which was used for signal transmission. A built-in PIC microcontroller was embedded in the transmission module, for the purpose of processing and data transmitting. Due to CLC100 sensor's output voltage exceeding the sensor board's input limitation, a voltage convertor was designed to connect the sensor and the wireless sensor board. The final results were voltages corresponding to the liquid level, and were processed by an independent PIC development board, and then sent to PC's hyper terminal via serial-port by this PIC microcontroller. Experiments showed that this wireless sensor node prototype worked well.

\section{Keywords}

CLC100 Liquid Level Sensor, Powercast Wireless Sensor Kit, Colorless Liquid Level Measurement, Wireless Sensor

\section{Background and Overview}

Liquid level is a key parameter existing in various industrial field or laboratory environments, and it's also a

How to cite this paper: Yan, G.L., Lindner, G. and Shen, Y.M. (2015) New Measuring Method for Colorless Liquid Level Based on CLC100 Level Sensor and Powercast Wireless Sensor Kit. Engineering, 7, 177-189. 
very important parameter for automatic control, so it's an important procedure to get liquid level quickly and accurately.

Situations like small range liquid level measurements, say for smaller than $100 \mathrm{~mm}$, need very accurate results and low measuring deviations. And if the measuring is by a chemical furnace or other dangerous places, direct measurement by people should be avoided for safety reason; And if online remote measurement is needed while processing data coming from other sensor nodes, direct measurement is also not available. If the liquid level measurement happens in such situations above, wireless sensor node is the best choice.

There are some widely used liquid level measurement methods. For example, ultrasonic level detection method [1], photoelectric detection, optical liquid level measurement method [2], magnetostrictive level measurement [2] and so on. Ultrasonic method is the most common way, but it gets easily to be disturbed by noises, and it can't be used in vacuum conditions [1]. Photoelectric detection is too critical to light sources, which means it can be easily affected by other light noise. So it's very hard to measure the level exposed in nature sunlight accurately. Magnetostrictive way has advantages like high accuracy, multiple signal output, easily adaptive to different environments [2]. However, magnetostrictive meter has to be contacted to the liquid while measuring, this could be a fatal disadvantage for caustic liquid.

Moreover, if the measured liquid is colorless, it will be more difficult, and will bring new problems for lightbased methods.

Recently, new type capacitive liquid level sensor CLC100 was invented for small range and critical measurement. It was chosen as the sensor part for the demo sensor node in this paper. Combined with Powercast's wireless sensor kit's wireless communication module, a new wireless measuring system is created.

\section{Introduction of Liquid Level Sensor}

CLC100 level sensor is the latest level measurement product from Sensortechnics Corporation in Germany in 2012 [3]. It uses the new contact-free capacitive sensor technology to measure the liquids level or the pulverized powers in different kinds of containers. It's easy to calibrate it and quite flexible to use it. It can be easily mounted outside of almost any non-metallic containers. It has a great application prospect, for example, chemical and pharmaceutical industries applications, medical technology field, biotechnology as well as food and beverage measurement.

Currently, this sensor is quite new that it hasn't been applied into many industrial fields, and there are few applications introduced on journals. MarcinTomasik and his colleagues in Agricultural University of Kraków in Poland wrote an article with respect to apply this sensor in autonomous milk measurement in milking units [3].

Detail information of CLC100 liquid level sensor is introduced here. The general appearance of this sensor is seen in Figure 1.

It has some unique features:

1) Contact-free measurement and can detect water level continuously. This characteristic is very helpful when the surface of container isn't so smooth. We don't need to stick the sensor tightly on the surface, instead, fix it parallel with the container's surface. But the total distance between the sensor and liquid should smaller than 5 mm. Standard mounting is shown in Figure 2.

2) It can measure not only liquid, but also solids like pulverized materials. Because CLC100 is capacitance sensitive element, powders can also be detected by affecting the working capacitance.

3) It also can be taken as a position sensor. Because displacement and object position can be changed into some liquid lever measurement, so CLC100 can also be used in small positon measurement applications.

4) It can measure almost any non-metallic material containers.

Key electrical parameters are seen in Table 1.

CLC100 can be mounted easily. Take a cylinder as example, schematic diagram is shown Figure 2 [4].

Before using this sensor, it should be mounted as required, and need to be calibrated. And calibration proce-

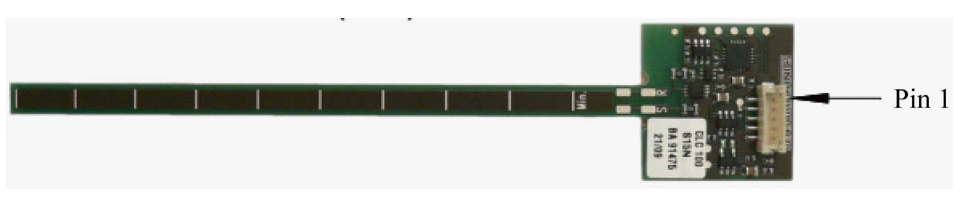

Figure 1. CLC100 liquid level sensor. 


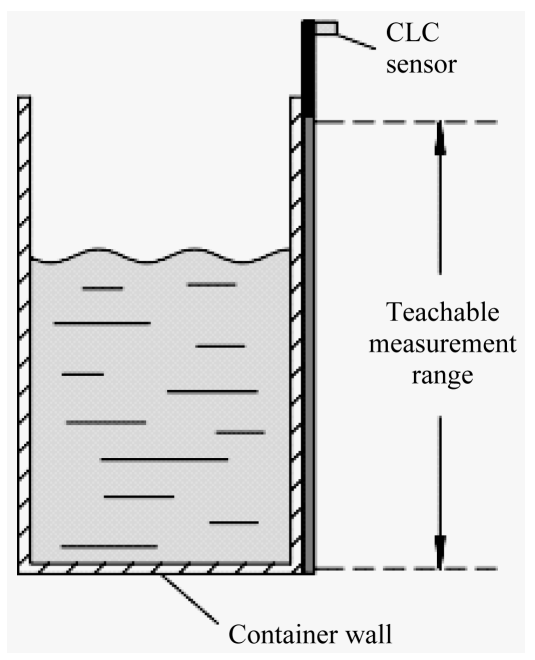

Figure 2. Mounting of CLC100.

\begin{tabular}{cc} 
Table 1. Electrical parameters of CLC100. \\
\hline Supply voltage $\left(\mathrm{V}_{\mathrm{s}}\right)$ & $5.5-15 \mathrm{~V}$, typically $9 \mathrm{~V}$ \\
Output voltage $\left(\mathrm{R}_{\mathrm{i}}=1 \mathrm{~K} \Omega\right)$ & $0.5-4.5 \mathrm{~V}$ \\
\hline
\end{tabular}

dures will be presented in experiments part.

CLC100 liquid level sensor can output two kinds of signals: analog voltage and digital hex numbers corresponding to the analog voltage. In this paper analog mode is used.

The measuring range of CLC100 is $0-100 \mathrm{~mm}$, and the corresponding analogy output voltage is $0.5-4.5 \mathrm{~V}$. The liquid level and voltage follow a linear relationship strictly. The relationship can be drawn like this (see Figure 3).

By the way, CLC100 sensors can be used in series, which means larger measurement range can be reached by using several sensor in series.

According to the datasheet of CLC100, 0\% - 100\% liquid level corresponds to single value between 0x01 $0 \mathrm{xEF}$, and also corresponds to the values between $0.5-4.5 \mathrm{~V}$. So the theoretical resolution is: $100 \mathrm{~mm} / 255=$ $0.392 \mathrm{~mm}$.

And according to the relation figure above, the analog output voltage is:

$$
\text { Analog voltage }(\mathrm{V})=0.04(\mathrm{~V} / \mathrm{mm}) \times \text { liquid level }(\mathrm{mm})+0.5
$$

The theoretical voltage resolution is $0.016 \mathrm{~V}$ approximately.

\section{Introduction of Powercast Wireless Sensor Kit}

Wireless sensor development kit is a wireless sensor development platform from PowerCast Corporation in United Stated. This kit is used for developing battery-free wireless sensor applications powered by RF energy (radio wave energy). The RF energy transmits radio frequency energy over distance using common radio waves.

Because radio waves have no certain direction, so any radio harvester in any the common radio field can get energy. It's very flexible. Besides, radio frequency energy stays reliable in the environment, not influenced by time, sunlight or weather. This makes the harvesting process easy to be controlled, and easier to be applied into real applications.

Since from 2007, a lot of patents about implementations of wireless power transmitter and receivers every year [5]. RF energy harvesting technology has been well studied in the past ten years, and according to Moore's law, the performance of RF energy harvesting technology could be doubled every two years [6]. And finally in year 2011, PowerCast developed this creative wireless development platform. It contains four parts: one radio frequency transmitter, two evaluation boards, two wireless sensor boards, one ultra-low power consumption (XLP) development board. 


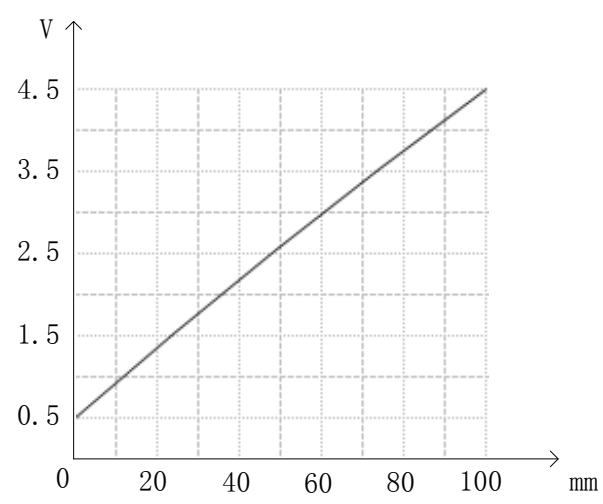

Figure 3. Corresponding relationship between voltage and liquid level.

\subsection{Radio Frequency Transmitter}

TX91501 Powercast Radio frequency Transmitter is the power source of the evaluation board and wireless sensor board (Figure 4). It is a box to transmit broadcast energy in distance. Maximum working distance is around $12 \mathrm{~m}$.

This transmitter is integrated with an $8 \mathrm{dBi}$ integrated antenna, and transmits $915 \mathrm{MHz}$ radio waves. The maximum transmitting power is set as 3 watt.

This RF energy transmitter is designed for low power consumption applications. Such as wireless battery charging, battery free wireless sensor nodes or wireless triggers.

Before this transmitter is used, receiving signal strength was tested within its effective working range, to make it clear which distance is the best for our wireless sensor node design. According to transmitter's datasheet, its working range is about 12 meters. 12 points were selected in its range unequally. When it's closer to the transmitter, more test points were tested, and less points were tested when it's farer to the transmitter.

The RF energy was harvested by the evaluation board with the default program, and $6 \mathrm{dBi}$ directional patch antenna was used to receive radio waves. Linear gain of this antenna is 4.1, energy pattern is: $122^{\circ}$ (horizontal), $68^{\circ}$ (vertical). The evaluation board will be explained in the next chapter.

Each point was tested 5 times, and average values were taken. Experiments results are shown in Table 2.

According to the experiments, highest conversion efficiency was reached at distance $1.5 \mathrm{~m}$. When it got closer or far away, the conversion efficiency would come down. So in the later experiments, the evaluation board for energy harvesting was always put at distance around 1.5 meters.

\subsection{Evaluation Board}

Evaluation board is the access point. It is used to receive RF energy and transfer it into electrical charge energy. This energy will drive evaluation board itself and the wireless sensor board also. See Figure 5 for the outlooking of evaluation board and its antennas.

There are two types of antennas, whose parameters are shown in Table 3.

In the whole experiments, patch antenna was used, because it had a bigger antenna gain, and could provide more energy at the same distance compared with dipole antenna.

P2110 power harvesting chip is the energy harvester receiver on evaluation board. Connected to antenna, it can convert RF energy into DC voltage. It has a high conversion efficiency and it can output voltage up to 5.25 $\mathrm{V}$ [7]. And it has a wide range RF operating range.

The converted DC Voltage is stored in capacitors. There are two integrated capacitors and one external interface for another customized capacitor. One of the three is selected according to the power of the sensor we use. For higher power consumption sensors connected to the wireless sensor board, bigger capacitor should be selected. When sufficient charge is stored, the P2110 provides the regulated output power to the evaluation board and the wireless sensor board until the sensor operation is completed, or until the power is turned off at the lowvoltage threshold on the capacitor. The threshold is around $2.2 \mathrm{~V}$.

The charging and discharging diagram can see from Figure 6. 
Table 2. Signal strength at different distance to the transmitter.

\begin{tabular}{cccc}
\hline Distance $(\mathrm{m})$ & Received signal strength $(\mathrm{mW})$ & Output of power harvester $(\mathrm{mW})$ & RF to DC conversion efficiency \\
\hline $0-0.3$ & 66.22 & Too short to be used & $48.0 \%$ \\
0.35 & 32.50 & 31.80 & $47.7 \%$ \\
0.50 & 16.51 & 15.50 & $47.7 \%$ \\
0.70 & 12.60 & 7.90 & $47.6 \%$ \\
0.80 & 8.10 & 6.02 & $50.6 \%$ \\
1.00 & 3.61 & 4.10 & $55.5 \%$ \\
1.50 & 1.30 & 2.00 & $50.8 \%$ \\
2.50 & 0.51 & 0.66 & $47.1 \%$ \\
4.00 & 0.23 & 0.24 & $47.8 \%$ \\
6.00 & 0.10 & 0.11 & $30.0 \%$ \\
9.00 & 0.06 & 0.03 & 0 \\
12.00 & & 0 & 0 Ot of range \\
$>12$ & & &
\end{tabular}

Note: The test experiments were done on open areas without any block stuff between the transmitter and the energy harvester, and the receiving antenna faced exactly to the transmitter. When it comes to real applications, if these conditions can't be met, less RF energy can be harvested.

\section{Table 3. Antenna information.}

\begin{tabular}{cccc}
\hline Name & Type & Energy pattern & Antenna gain \\
\hline Dipole antenna & Omni-directional vertical polarized & $360^{\circ}$ & $1.25(1.0 \mathrm{dBi})$ \\
Patch antenna & Directional vertical polarized & $122^{\circ}$ (horizontal) $68^{\circ}$ (vertical) & $4.1(6.1 \mathrm{dBi})$ \\
\hline
\end{tabular}

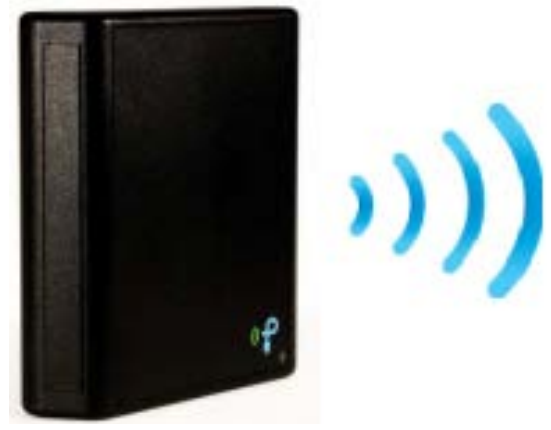

Figure 4. Power transmitter.
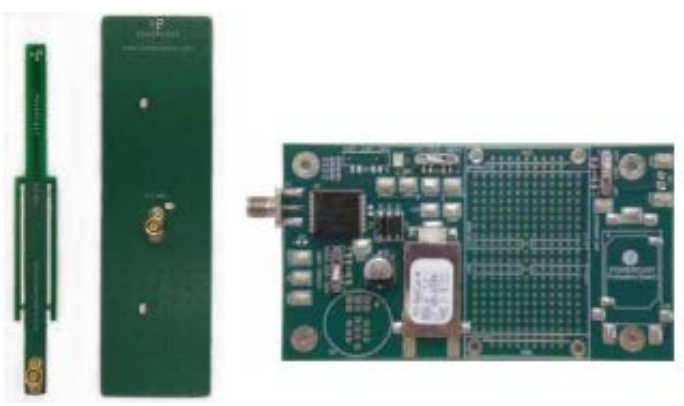

Figure 5. Evaluation board and its antennas. 
If bigger capacitor is used, it will be charged slower but provide longer operation time for the evaluation board; smaller capacitor will be charged quicker and can provide shorter operation time, and it may not enough to drive the whole evaluation board and the sensor board. The required capacitor can be calculated from the following equation:

$$
C=15 V_{\text {OUT }} I_{\text {OUT }} t_{\text {ON }}
$$

$V_{\text {OUT }}$ : Output voltage of chip P2110;

$I_{\text {OUT }}$ : Average output current from chip P2110;

$t_{\text {ON }}$ : Operation time of evaluation board.

P2110 energy harvesting chip can be applied in battery free wireless sensors, like the evaluation board and the wireless sensor board used in this paper. It can also be applied in other applications like wireless trigger or other electronic units. Here is a typical application circuit, see it from Figure 7.

\subsection{Wireless Sensor Board}

Wireless sensor board is the attached board for evaluation board. The working power is also provided by the P2110 energy harvesting chip. This board is composed of four main parts: built-in PIC microcontroller, wireless communication antenna, built-in sensors and one extended voltage interface for reading extended sensor's output voltage. Also the interface can also provide $3 \mathrm{~V}$ voltage supply to the extended sensor if it is low-power consumption sensor, but this voltage can't be adjusted like evaluation board. Due to this reason, extra power supply was provided for the liquid level sensor. This part could be solved when other suitable lower-voltage supplied sensors is applied instead.

The general appearance of this wireless sensor board can see from Figure 8 [8].

The antenna module is a combination of a MRF24J40 communication chip and one antenna (the left part in Figure 8). The built-in sensors include one thermistor temperature sensor NCP18XH103F03RB; one light in-

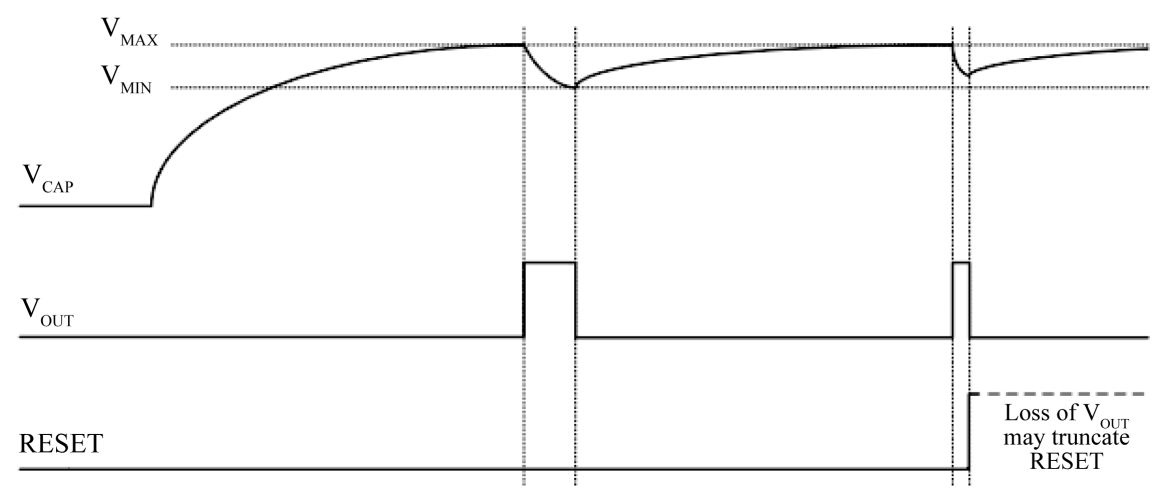

Figure 6. Charging and discharging diagram.

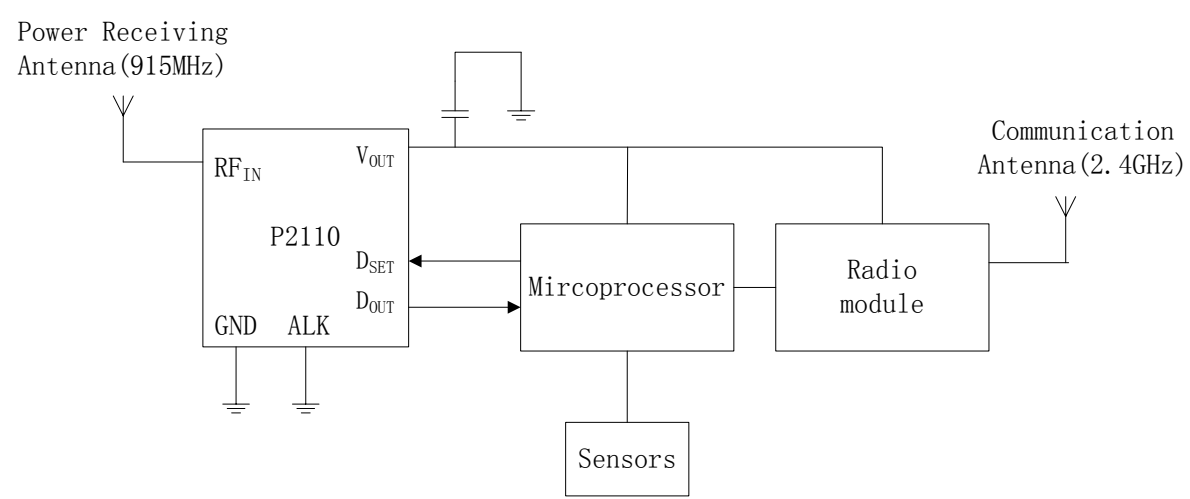

Figure 7. Typical application circuit of power harvesting. 


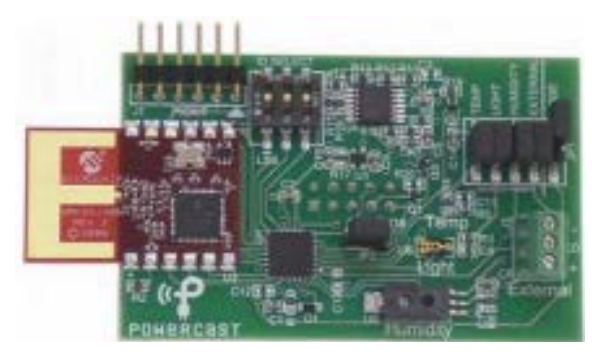

Figure 8. Wireless sensor board.

tensity sensor TEMT6000X01; one humidity sensor HIH-5030. The extended interface was mainly used in the paper experiments for developing new wireless sensor prototype. But they were not used in this liquid level measurement design. Extended analog voltage-reading port was used for our liquid level sensor. This board also has a built-in analog to digital convertor, and the A/D conversion and the wireless communication are controlled and optimized by a built-in PIC microcontroller.

The communication chip is MRF24J40MA, which is a complete 2.4 GHz IEEE 802.15.4 compliant surface mount module. It has a small size of $17.8 \mathrm{~mm} \times 27.9 \mathrm{~mm}$, low current consumption of $19 \mathrm{~mA}$ when working and receiver, and $23 \mathrm{~mA}$ when working as transmitter. And in sleep mode, it comes down to $2 \mu \mathrm{A}$. Its operation Band is $2.40-2.48 \mathrm{GHz}$, and the communication rate is $250 \mathrm{kbps}$. Besides, it is integrated with targeted crystal, internal voltage regulator, matching circuitry and one communication antenna [9].

MRF24J40 communicates with PIC controllers via a four-line SPI bus, and it can be directly connected to PIC microchip, because there is an integrated SPI module inside. The interface of MRF24J40 and PIC24F16 microcontroller is shown in Figure 9.

These chips can communicate with each other at distance around 120 meters. For designing wireless sensor communications in this paper, this working range is enough.

Other features of MRF24J40 communication chips see below [9]:

- Integrated Low Phase Noise VCO, Frequency Synthesizer and PLL Loop Filter;

- Digital VCO and Filter Calibration;

- Integrated RSSI ADC and I/Q DACs;

- High Receiver and RSSI Dynamic Range MAC/Baseband Features;

- Hardware CSMA-CA Mechanism, Automatic ACK Response and FCS Check;

- Supports all CCA modes and RSS/LQI;

- Automatic Packet Retransmit Capable;

- Hardware Security Engine (AES-128) with CTR, CCM and CBC-MAC modes;

- Supports Encryption and Decryption for MAC Sub-layer and Upper Layer.

\subsection{XLP Development Board}

Ultra-low power consumption (XLP) development is a universal PIC microcontroller board originally from Microchip, and is included in this wireless sensor development platform. The main units on this board are a PIC24F16KA102 microcontroller and its basic peripherals. It's the control board of signal receiving and data displaying. One access point (signal receiving board based on MRF24J40 module) is matched with this development board by a 28 pins interface.

The general appearance of the control board and wireless its access point are seen in Figure 10. It works under 3.3 V voltage, and be powered through a USB cable or by two dry cells.

The red access point is a communication MRF24J40 module with an antenna. This module is the same with the module integrated in wireless sensor board. One performs as transmitter and the other works as receiver.

This development board consists of a PIC microcontroller development board and one PIC tail board. In each part the PIC, controllers are PIC24F16KA102, but with different package. The tail board is an antenna board used for receiving signals coming from the sensor board.

\section{Wireless Liquid Level Sensor Implementation}

The general frame of this wireless liquid level sensor implementation can be seen in Figure 11. 


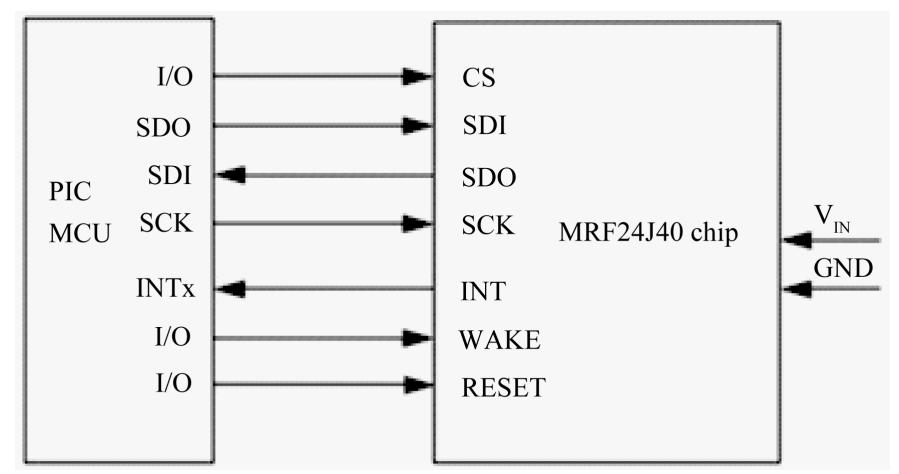

Figure 9. The interface between PIC24F16 and MRF24J40.
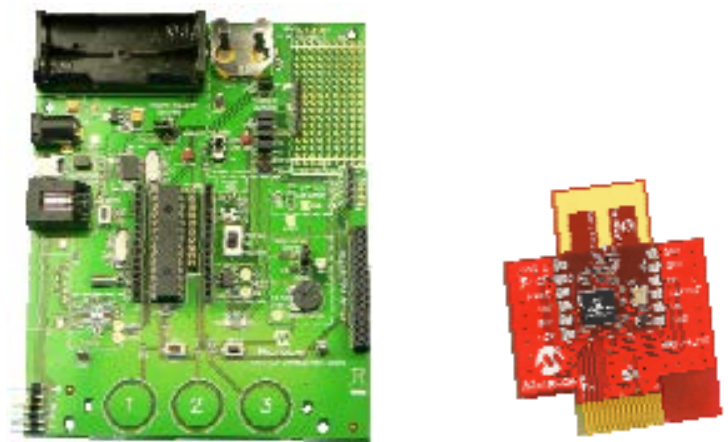

Figure 10. XLP development board and its access point.

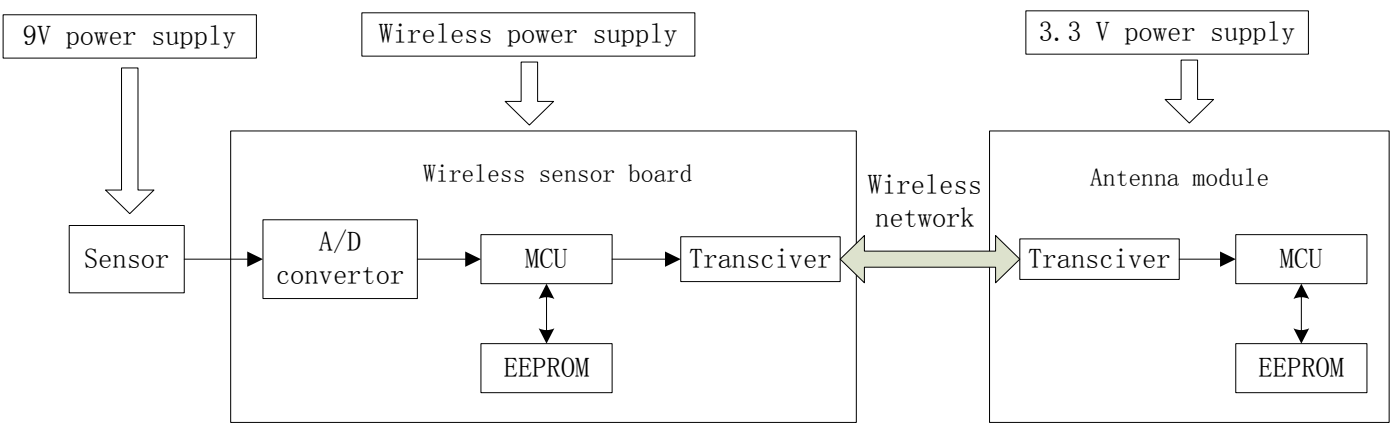

Figure 11. Structure of sensor node.

In this paper, Microprocessor is PIC24F16KA102;

The components are as follows:

Sensor used: CLC100 liquid level sensor;

Transceiver: two MRF24J40 modules;

Power supply: wireless sensor board is powered by its RF energy transmitter. But due to the liquid level sensor we used in the experiment need higher voltage supply, so an independent $9 \mathrm{~V}$ voltage was supplied additionally. This could be changed while other lower power consumption liquid level sensor is applied.

The wireless sensor board itself has an analog extended port for reading external sensors' output. The reading voltage range is: 0 - $3000 \mathrm{mV}$, and this value will be processed by the built-in microcontroller, and be sent out by the built-in antenna. But the output range of CLC 100 sensor is: $0.5 \mathrm{~V}-4.5 \mathrm{~V}$, so voltage convertor need to be designed.

\subsection{DC-DC Conversion Circuit}

The goal was to convert voltage range $0.5-4.5 \mathrm{~V}$ into range $0-3 \mathrm{~V}$. The converting thinking consisted of two 
parts: first, using a subtractor circuit to decrease the sensor's output voltage to range 0 - $4 \mathrm{~V}$; second, using a reverse amplifier to decrease the voltage range again into $0-3 \mathrm{~V}$. The amplification was set to 0.75 according this requirement. The implementation circuit is shown in Figure 12.

LM324 was a widely used amplifier in electronic designs, and it was adopt in this circuit as well. LM324 could work under large voltage range: 3 - $32 \mathrm{~V}$, and it worked well under dual power supply. In our experiments, $\pm 5 \mathrm{~V}$ was provided. Besides, 6 resistors were used in the design, their values are shown in Table 4.

This DC-DC conversion circuit has two parts. The left half part is subtractor circuit. $0.5 \mathrm{~V}$ is the provided as reference voltage, and will be subtracted by every input voltage in real-time. Because the minimum voltage we can get from CLC100 liquid level sensor is $0.5 \mathrm{~V}$, so a negative value will be get after this subtraction. The right half, starting from resistor R5 to the end, is the amplifier circuit with 0.75 times amplification. Each part has a voltage meter, monitoring the correctness of output.

At the upper-left corner, a cell batter is used for simulating the sensor's output voltage, and in the circuit pasted here, $4.5 \mathrm{~V}$ is set, which is the maximum output of CLC100 sensor. The rightest part of this circuit is the DC-DC convertor's output. When CLC100 output reaches its maximum measuring level (HIGH value), it outputs $4.5 \mathrm{~V}$ through its analog pin, and here $4.5 \mathrm{~V}$ battery is the replacement in Proteus. $-4 \mathrm{~V}$ and $3 \mathrm{~V}$ can be read from the two voltage meters. $3 \mathrm{~V}$ is the final voltage we need, and it maximum value need to be converted. Results here show that this conversion circuit works well. And of course the whole voltage range, $0.5-4.5 \mathrm{~V}$, was tested and verified by step of $0.1 \mathrm{~V}$ ascendingly from $0 \mathrm{~V}$ in Proteus. Simulation showed that this conversion circuit worked very well on our required voltage conversion purpose.

The real circuit was also built on bread board, and was tested as well. Bread board implementation seen as Figure 13.

The $\pm 5 \mathrm{~V}, 0.5 \mathrm{~V}$, GND, circuit input (sensor's output) and the DC-DC output are marked in the figure.

\subsection{Implementation Frame of Wireless Liquid Level Sensor}

After the voltage convertor is designed, CLC100 can be connected to the wireless sensor board through it. Liquid level sensor, voltage convertor and the wireless sensor board make up a wireless liquid level sensor node. All kinds of liquid level can be measured by this node and data will be transmitted through the built-in antenna.

The sensor node construction frame is shown in Figure 14.

We can see that, the built-in A/D convertor, one PIC microcontroller and one wireless module are the main components of our wireless sensor board.

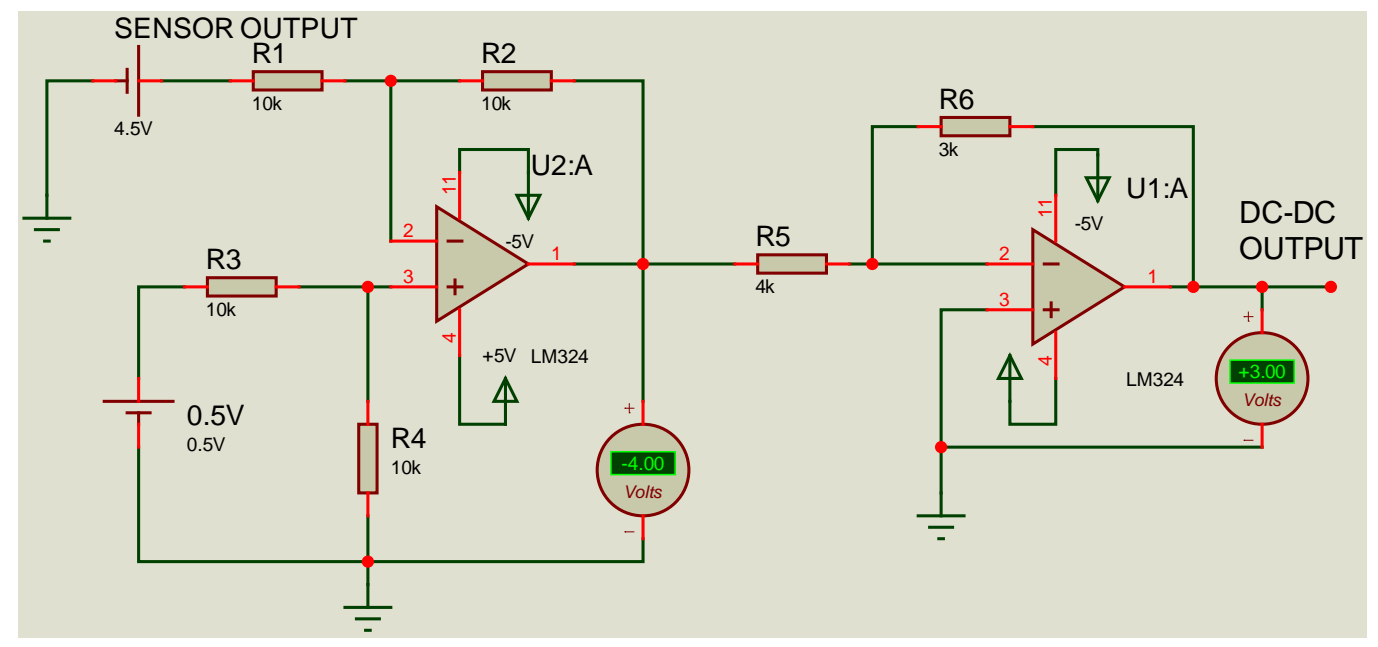

Figure 12. DC-DC conversion circuit.

Table 4. Resistors used in the conversion circuit.

\begin{tabular}{ccccccc}
\hline Resistor & $\mathrm{R} 1$ & $\mathrm{R} 2$ & $\mathrm{R} 3$ & $\mathrm{R} 4$ & $\mathrm{R} 5$ & $\mathrm{R} 6$ \\
Resistance & $10 \mathrm{~K}$ & $10 \mathrm{~K}$ & $10 \mathrm{~K}$ & $10 \mathrm{~K}$ & $4 \mathrm{~K}$ & \\
\hline
\end{tabular}




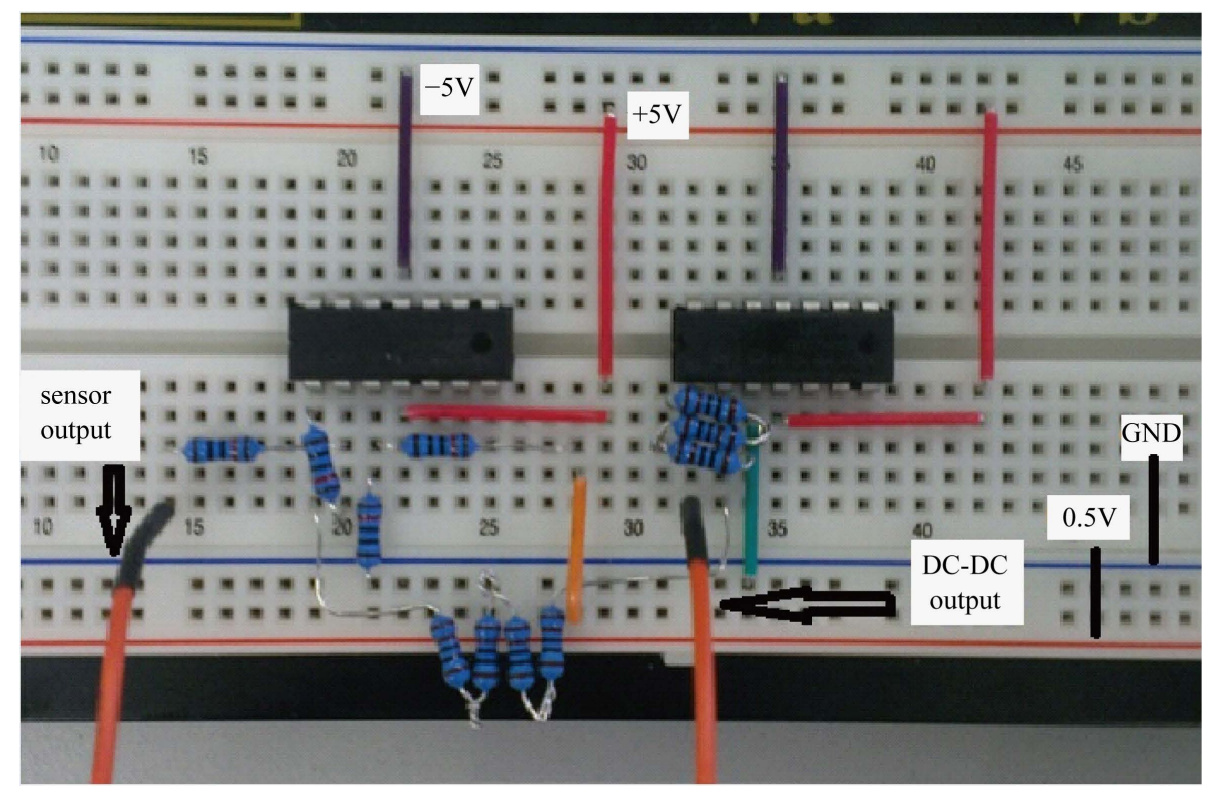

Figure 13. Beard board implementation of conversion circuit.

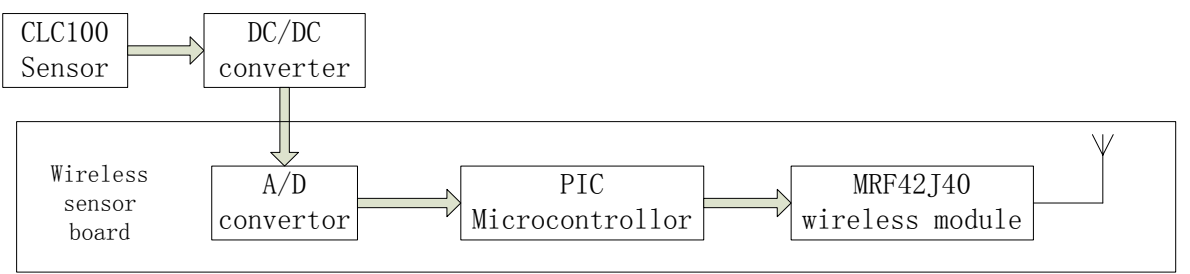

Figure 14. Construction frame of liquid level sensor node.

When data is transmitted out from wireless liquid level sensor node, it comes to the data receiving antenna. This antenna module is the same with the data transmitting module in construction. Data will be captured by the PIC microcontroller in XLP development board, and then the data will be sent to PC, and displayed in a hyper terminal.

Data flow and the hardware modules are shown in Figure 15.

XLP: Ultra Low Power Consumption.

\subsection{Experiments and Discussions}

\subsubsection{Experiments}

Normal glass cup is chosen as the container, filled with tap water as liquid. The mounting of CLC100 and the measuring setup is shown in Figure 16.

CLC100 liquid level sensor should be calibrated before used. The Calibration is called teach-in [4]. Calibration is to tell the sensor the measuring range: the lowest water level (LOW value) and the highest water level (HIGH value). Water level below the lowest level will be determined as the LOW value, and water level higher than the highest will be determined as HIGH value. The two values will be stored in the built-in $\mathrm{E}^{2} \mathrm{PROM}$.

The calibration steps are as follows:

1) Connect sensor to $+9 \mathrm{~V}$ power supply ( $V_{S}$ pin) and ground (GND pin).

2) Wait for 2 seconds for a successful start-up.

3) Calibrate LOW value first: connect the teach-in pin (TI) to voltage $+0.3 \mathrm{~V}$.

4) Wait for 1.5 seconds.

5) Calibrate HIGH value then: connect the TI pin to voltage $4.8 \mathrm{~V}$.

6) Wait for 1.5 seconds.

After the steps above, two LOW and HIGH value will be stored in the built-in E ${ }^{2}$ PROM, and they can be reset by the next teach-in process. For one application, only one calibration is needed. When the sensor is used for 


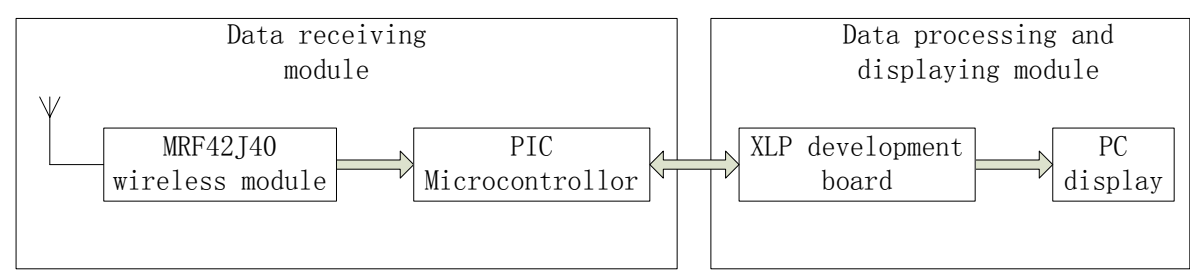

Figure 15. Date receiving and processing module.

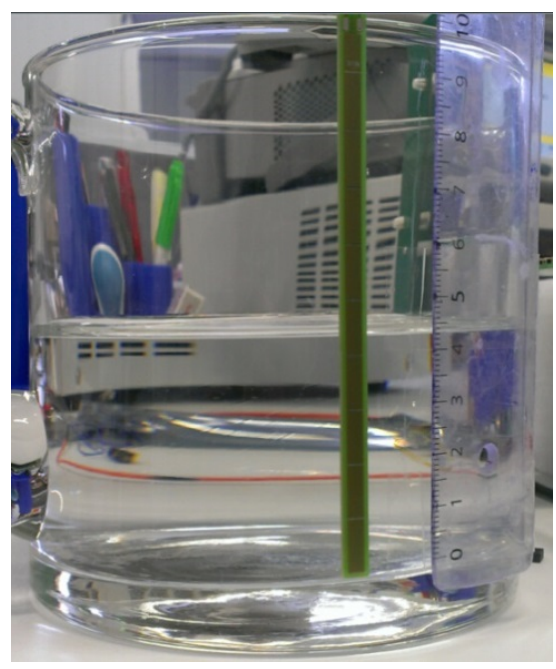

Figure 16. Image of measuring setup.

another container's liquid level measurement, new calibration should be made, following the same procedures. In this paper's experiments, LOW value and HIGH value were set to $0 \mathrm{~mm}$ and $100 \mathrm{~mm}$ by a ruler.

During experiments, 6 points are chosen between LOW level and HIGH level (these two points are included). 5-times repetitive experiments were made, and average values were calculated. The relationship between output voltage and water level is listed in Table 5 . Theoretical voltages were also calculated for comparison purpose.

Put the 6 value points into the voltage-liquid level chart, shown Figure 17.

From the above table and chart, we can see deviation existed in the experiment. There could be two reasons: first, because the liquid level sensor need be calibrated before used, and in this experiment, the normal $20 \mathrm{~cm}$ long ruler ( $0.5 \mathrm{~mm}$ resolution) was used, and this calibration could lead to a small deviation. Second, both the sensor output signal and the sampled signal by sampling board were analog signal, and they could be influenced by electrical noise in the experimental environment, like, power supply box. Besides, the sampling board and the development board used different ground connections, and this could also be a reason.

\subsubsection{Results Display Interface}

Voltage outputs are transferred wirelessly to the data-processing microcontroller, and then transferred to PC and displayed in hyper terminal. "Extrnl” value (in the red rectangles) stands for out analog voltage value. The display interface is shown in Figure 18.

In these experiments, there existed $20 \mathrm{mV}$ noise, which means $0.4 \mathrm{~mm}$ deviation in liquid level measurement.

\section{Conclusions}

In this paper, a new wireless measuring system for colorless liquid level measurement is designed. This new measuring system is based on the latest products: CLC100 liquid level sensor and Powercast wireless sensor kit.

The whole system is divided into two parts, level measurement and data transmission part (WSN part), data receiving and display part. First part includes a new type capacitive liquid level sensor CLC100 and the wireless senor sampling board. The CLC100 is used for liquid level measurement; the wireless sensor board is used for signal transmission, which is based on core communication chip MRF2J40. A PIC built-in microcontroller is 
Table 5. Experiments results.

\begin{tabular}{ccccccc}
\hline Water level (mm) & 0.0 & 20.0 & 40.0 & 60.0 & 80.0 & 100.0 \\
Theoretical voltage (V) & 0.500 & 1.300 & 2.100 & 2.900 & 3.700 & 4.500 \\
Real voltage (V) & 0.542 & 1.332 & 2.241 & 3.057 & 3.827 & 4.519 \\
\hline
\end{tabular}

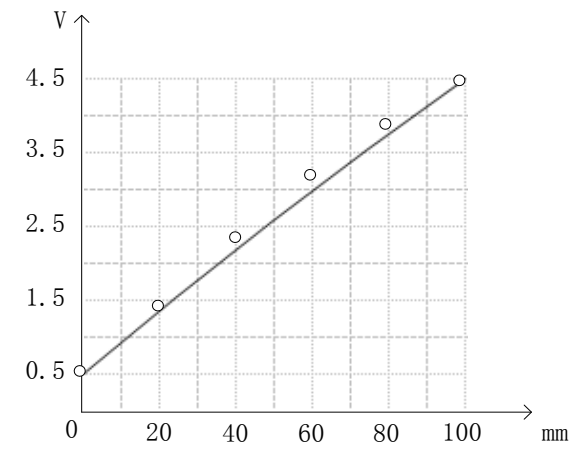

Figure 17. Comparison of theoretical values and real values.

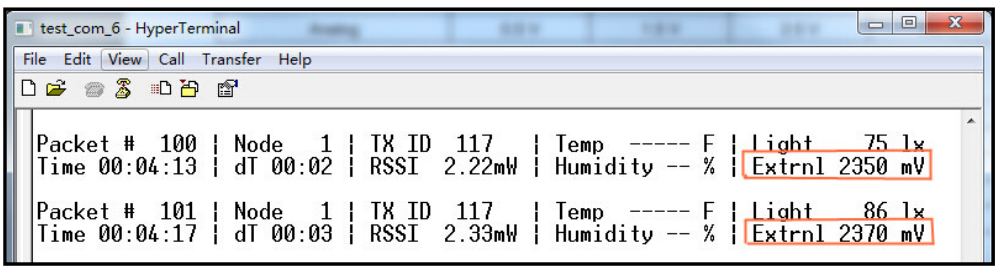

Figure 18. Display interface of voltage.

embedded in the transmission module, for the purpose of processing and transmitting data.

Due to sensor's output voltage exceeding the sensor board's input limitation, a voltage convertor is designed to connect the sensor and the wireless sensor board. The final results are voltages corresponding to the liquid level, and are processed by an independent PIC development board, and then sent to PC's hyper terminal via serial-port by this PIC microcontroller. Experiments show that this wireless sensor node prototype worked well.

But there are still problems need to be solved. For example, the liquid level sensor we used is not lowpower consumption sensor, thus an extra power supply is need on measuring site. In the future, this sensor could be replaced by other low-power consumption liquid level sensor. And the values displayed on screen are voltage values, which haven't been converted into physical level units. In later studying works, this should be done.

\section{References}

[1] Yuan, X.P. and Chen, Y. (2009) Commonly Used Research Methods in Liquid Level Detection. Energy Technology and Management, 1, 120-122.

[2] Yang, Z.H. and Li, H. (2009) Situation and Development Trend of New Liquid Level Detection Technology. Industry and Mine Automation, 6, 61-64.

[3] Tomasik, M., Juszka, H. and Lis, S. (2012) Communication System Control and Measurement System in the Autonomous Milking Machine. Inżynieria Rolnicza, 16, 303-310.

[4] CLC Series Miniature Capacitive Continuous Liquid Level Sensors Datasheet. Sensor Technics. www.sensortechnics.com

[5] Greene, C.E., Harrist, D.W. and McElhinny, M.T. (2007) Implementation of a Wireless Power Transmitter and Method. US Patent. 
[6] Shearer, J.G., Greene, C.E. and Harrist, D.W. (2010) Power Transmission Network. US Patent.

[7] Datasheet, P. (2011) P2110-915MHz RF Power Harvester Receiver. Powercast Corporation.

[8] User Manual of Lifetime Power Energy Harvesting Development Kit for Wireless Sensors. www.powercastco.com

[9] (2008) MRF24J40MA Data Sheet. Mircochip Technology Inc. 